Appl. Set-Valued Anal. Optim. 1 (2019), No. 2, pp. 135-147

Available online at http://asvao.biemdas.com

https://doi.org/10.23952/asvao.1.2019.2.04

\title{
HYBRID ITERATIVE METHODS FOR MULTIPLE SETS SPLIT FEASIBILITY PROBLEMS
}

\author{
QINGQING CHENG ${ }^{1}$, REKHA SRIVASTAVA ${ }^{2}$, QING YUAN ${ }^{3, *}$ \\ ${ }^{1}$ Department of Science, Tianjin University of Commerce, Tianjin 300134, China \\ ${ }^{2}$ Department of Mathematics and Statistics, University of Victoria, Victoria V8W 3R4, British Columbia, Canada \\ ${ }^{3}$ School of Mathematics and Statistics, Linyi University, Linyi 276000, China
}

\begin{abstract}
Based on viscosity techniques, we propose two hybrid iterative methods for a multiple-sets split feasibility problem. Under appropriate conditions, we establish two strong convergence theorems in a Hilbert spaces.

Keywords. Multiple-sets split feasibility problems; Split feasibility problem; Iterative method; Viscosity approximation method; Fixed point.
\end{abstract}

2010 Mathematics Subject Classification. 47H05, 47H09, 47J25.

\section{INTRODUCTION}

The split feasibility problem (SFP), which was proposed by Censor and Elfving [1], is to find

$$
x \in C \text { such that } A x \in Q \text {, }
$$

where $A: H_{1} \rightarrow H_{2}$ be a bounded linear operator, $C$ and $Q$ are nonempty closed convex subsets of real Hilbert spaces $H_{1}$ and $H_{2}$, respectively. This problem arises in signal processing, image reconstruction, and many other important applied fields. A number of image reconstruction problems can be formulated as the SFP and many iterative algorithms have been introduced to solve the SFP; see $[2,3,4,5,6,7,8$, $9,10,11]$ and references therein.

In [1], Censor and Elfving used multidistance ideas to study the SFP. Their algorithms involve matrix inverses at each iteration. In [2], Byrne presented a projection method called the CQ algorithm for solving the SFP that does not involve matrix inverses as follows:

$$
x_{n+1}=P_{C}\left[I-\gamma A^{*}\left(I-P_{Q}\right) A\right] x_{n}, \forall x_{0} \in H,
$$

where $0<\gamma<\frac{2}{\rho\left(A^{*} A\right)}, \rho\left(A^{*} A\right)$ is the spectral radius of $A^{*} A$.

In 2010, Xu [12] further studied the CQ algorithm and its convergence via fixed point methods. Xu [12], and Qin and Yao [10] proved that the problem is equivalent to a fixed point problem of the operator $P_{C}\left[I-\gamma A^{*}\left(I-P_{Q}\right) A\right]$. They proved that a point $x^{*}$ solves SFP if and only if $x^{*}=P_{C}\left[I-\gamma A^{*}\left(I-P_{Q}\right) A\right]$. Mann's iterative method have been applied to solve the SFP. However, Mann's method is only weakly

\footnotetext{
${ }^{*}$ Corresponding author.

E-mail addresses: chengqingqing2006@126.com (Q. Cheng), yuanqing@lyu.edu.cn (Q. Yuan), rekhas@ math.uvic.ca (R. Srivastava).

Received June 7, 2019; Accepted July 16, 2019.
}

(C)2019 Applied Set-Valued Analysis and Optimization 
convergent in an infinite dimensional space. Indeed, strong convergence is more important in many engineering fields. To obtain strong convergence theorems, Sitthithakerngkiet et al. [13] studied the following fixed point algorithm for the SFP

$$
x_{n+1}=\alpha_{n} \gamma f\left(x_{n}\right)+\left(I-\alpha_{n} B\right) P_{C}\left[I-\gamma A^{*}\left(I-P_{Q}\right) A\right] x_{n}
$$

where $f$ is a contraction on $H$ and $B$ is a strongly positive bounded linear self-adjoint operator on $H$ with coefficient $\bar{\gamma}>0, \alpha_{n} \subset(0,1)$ is a slowly vanishing sequence and $\gamma>0$ is a constant. Under appropriate conditions, they proved $\left\{x_{n}\right\}$ converges strongly to a point $x^{*} \in \Gamma$, which is also the unique solution of some monotone variational inequality. As an extension of the split feasibility problem, the multiple-sets split feasibility problem (MSFP), which was recently introduced [14], is formulated as finding a point $x$ with the property:

$$
x \in \bigcap_{i=1}^{N} C_{i} \text { and } A x \in \bigcap_{j=1}^{M} Q_{j} .
$$

The MSSFP (1.1) with $N=M=1$ is the split feasibility problem. The multiple-sets split feasibility problem arises in many practical fields, such as, image reconstruction, signal processing, intensity-modulated radiation therapy (IMRT) and so on. Recently, the MSFP received much attention and many researchers proposed fixed point algorithms for solving it; see, [11, 15, 16, 17, 18, 19] and references therein.

As a direct extension of the CQ algorithm, Wang and $\mathrm{Xu}$ [20] gave a cyclic algorithm to solve the MSSFP:

$$
x_{n+1}=P_{C_{[n]}}\left[I-\gamma A^{*}\left(I-P_{Q_{[n]}}\right) A\right] x_{n}
$$

where $[n]:=n(\bmod p),(\bmod$ function take values in $\{1,2, \cdots, p\})$. They showed that the sequence $\left\{x_{n}\right\}$ convergence weakly to a solution of then MSSFP whenever its solution set is nonempty.

In [21], Tang and Liu proposed simultaneous and cyclic iterative algorithms for solving a split common fixed point problem and applied their main results to the multiple-sets split feasibility problem. Up to our knowledge, many weak convergence theorems of solutions were established in Hilbert spaces and Banach spaces. In many subjects, strong convergence is more applicable.

Motivated by the above related results in this field, we propose two hybrid iterative method for solving the multiple-set split feasibility problem and establish two strong convergence theorems. Our solution also uniquely solve some monotone variational inequality. The rest of the paper is organized as follows. In Section 2, we give some basic definitions, propositions and lemmas. In Section 3, we presents our hybrid iterative methods to solve the MSSFP and obtain strong convergence theorems of solutions.

\section{PRELIMINARIES}

Throughout the paper, let $H_{1}$ and $H_{2}$ be an infinite dimensional real Hilbert space with inner product and norm denoted by $\langle\cdot, \cdot\rangle$ and $\|\cdot\| . \rightarrow$ and $\rightarrow$ denote the strong convergence and weak convergence, respectively. In addition, $F(T)$ and $\omega_{w}\left(x_{n}\right)$ denote the fixed point set of $T$ and the weak $\omega$-limit set of the sequence $\left\{x_{n}\right\}$, respectively, that is, $F(T)=\{x: T x=x\}$ and $\omega_{w}\left(x_{n}\right)=\left\{u: \exists x_{n_{j}} \rightarrow u\right\}$. Below we gather some basic definitions and results which are needed in the subsequent section.

Recall that a mapping $T: H \rightarrow H$ is said to be $\rho$-Lipschitzian with $\rho>0$ if

$$
\|T x-T y\| \leq \rho\|x-y\|, \quad \forall x, y \in H .
$$

If $0<\rho<1$, then $T$ is a $\rho$-contraction. If $\rho=1$, then $T$ is a nonexpansive mapping. 
Given a nonlinear mapping $F: C \rightarrow H$. Recall that $F$ is said to be monotone if

$$
\langle x-y, F x-F y\rangle \geq 0, \quad \forall x, y \in C .
$$

$B$ is said to be $\alpha$-strongly monotone if there exists $\alpha>0$ such that

$$
\langle x-y, F x-F y\rangle \geq \alpha\|x-y\|^{2}, \quad \forall x, y \in C .
$$

$B$ is said to be $\alpha$-inverse strongly monotone (for short, $\alpha$-ism) if there exists $\alpha>0$ such that

$$
\langle x-y, F x-F y\rangle \geq \alpha\|F x-F y\|^{2}, \quad \forall x, y \in C .
$$

We can easily see that

(i) if $F$ is nonexpansive, then $I-F$ is monotone;

(ii) if $F$ is an $\alpha$-inverse-strongly monotone mapping, then it must be $\frac{1}{\alpha}$-Lipschitz. Moreover, $I-r F$ is nonexpansive when $0<r \leq 2 \alpha$.

Recall that $P_{C}$ is the metric projection from $H$ into $C$. Then for each point $x \in H$, the unique point $P_{C} x \in C$ satisfies the property:

$$
\left\|x-P_{C} x\right\|=\inf _{y \in C}\|x-y\|=: d(x, C) .
$$

Lemma 2.1. ([22]) For a given $x \in H$, we have

(i) $z=P_{C} x$ if and only if $\langle x-z, z-y\rangle \geq 0, \forall y \in C$;

(ii) $z=P_{C} x$ if and only if $\|x-z\|^{2} \leq\|x-y\|^{2}-\|y-z\|^{2}$;

(iii) $\left\langle P_{C} x-P_{C} y, x-y\right\rangle \geq\left\|P_{C} x-P_{C} y\right\|^{2}, \forall x, y \in H$.

It is obvious that $P_{C}$ is nonexpansive and monotone.

Lemma 2.2. (Demiclosedness principle, [23]) Let $T: C \rightarrow C$ be a nonexpansive mapping with Fix $(T) \neq$ $\emptyset$. If $\left\{x_{n}\right\}$ is a sequence in $C$ that converges weakly to $x$ and $\left\{(I-T) x_{n}\right\}$ converges strongly to $y$, then $(I-T) x=y$. In particular, if $y=0$, then $x \in$ Fix $(T)$.

Lemma 2.3. ([24]) Let $C$ be a nonempty closed convex subset of a strictly convex Banach space X. Let $S_{1}$ and $S_{2}$ be two nonexpansivemappings from $C$ into itself with a common fixed point. Define a mapping $S: C \rightarrow C$ by

$$
S x=\delta S_{1} x+(1-\delta) S_{2} x, \forall x \in C,
$$

where $\delta$ is a constant in $(0,1)$. Then $S$ is nonexpansive and $F(S)=F\left(S_{1}\right) \cap F\left(S_{2}\right)$.

Recall that $T: H \rightarrow H$ is said to be firmly nonexpansive if $2 T-I$ is nonexpansive, or equivalently,

$$
\langle x-y, T x-T y\rangle \geq\|T x-T y\|^{2}, \quad x, y \in H .
$$

Alternatively, $T$ is firmly nonexpansive if and only if $T$ can be expressed as

$$
T=\frac{I+S}{2},
$$

where $S: H \rightarrow H$ is nonexpansive. 
Definition 2.1. A mapping $T: H \rightarrow H$ is said to be an averaged mapping if it can be written as the average of the identity $I$ and a nonexpansive mapping, that is,

$$
T=(1-\alpha) I+\alpha S
$$

where $\alpha \in(0,1)$ and $S: H \rightarrow H$ is nonexpansive. More precisely, if (2.1) holds, we say that $T$ is $\alpha$ averaged (for short, $\alpha$-av).

Clearly, a firmly nonexpansive mapping (in particular, a projection) is $\frac{1}{2}$-averaged.

Proposition 2.1. (Basic properties of averaged mappings, [3]) Let $S, T$ and $V$ be mappings on $H$. Then

(i) if $T=(1-\alpha) S+\alpha V$, where $\alpha \in(0,1)$, $S$ is averaged and $V$ is nonexpansive, then $T$ is averaged;

(ii) $T$ is firmly nonexpansive if and only if the complement $I-T$ is firmly nonexpansive;

(iii) If $T=(1-\alpha) S+\alpha V$, where $\alpha \in(0,1)$, $S$ is firmly nonexpansive and $V$ is nonexpansive, then $T$ is averaged;

(iv) the composite of finitely many averaged mappings is averaged. That is, if each of the mappings $\left\{T_{i}\right\}_{i=1}^{N}$ is averaged, then so is the composite $T_{1} \ldots T_{N}$. In particular, if $T_{1}$ is $\alpha_{1}$-averaged and $T_{2}$ is $\alpha_{2}$-averaged, where $\alpha_{1}, \alpha_{2} \in(0,1)$, then the composite $T_{1} T_{2}$ is $\alpha$-averaged, where $\alpha=$ $\alpha_{1}+\alpha_{2}-\alpha_{1} \alpha_{2}$

(v) if $\left\{T_{i}\right\}_{i=1}^{N}$ are averaged and have a common fixed point, then $\bigcap_{i=1}^{N} \operatorname{Fix}\left(T_{i}\right)=F i x\left(T_{1} \ldots T_{N}\right)$;

(vi) if $T$ is $\alpha$-averaged, then

$$
\|T x-z\|^{2} \leq\|x-z\|^{2}-\frac{1-\alpha}{\alpha}\|T x-x\|^{2}, x \in H, z \in F i x(T) .
$$

The following proposition summarizes some results on the relations between averaged mappings and inverse strongly monotone operators.

Proposition 2.2. ([3]) Let $T: H \rightarrow H$ be a mapping. Then

(i) $T$ is nonexpansive if and only if the complement $I-T$ is $\frac{1}{2}-i s m$;

(ii) if $T$ is $v$-ism, then for $\gamma>0, \gamma T$ is $\frac{v}{\gamma}$-ism;

(iii) $T$ is averaged if and only if the complement $I-T$ is $v$-ism for some $v>\frac{1}{2}$. Indeed, for $\alpha \in(0,1)$, $T$ is $\alpha$-averaged if and only if $I-T$ is $\frac{1}{2 \alpha}$-ism.

Lemma 2.4. ([25]) Let $H_{1}$ and $H_{2}$ be two real Hilbert spaces. Let $A: H_{1} \rightarrow H_{2}$ be a bounded linear operator, and let $A^{*}$ be the adjoint of $A$. Let $C$ be a nonempty closed convex subset of $H_{2}$, and let $G: H_{2} \rightarrow H_{2}$ be a firmly nonexpansive mapping. Then $A^{*}(I-G) A$ is a $\frac{1}{\|A\|^{2}}-i s m$, that is,

$$
\frac{1}{\|A\|^{2}}\left\|A^{*}(I-G) A x-A^{*}(I-G) A y\right\|^{2} \leq\left\langle x-y, A^{*}(I-G) A x-A^{*}(I-G) A y\right\rangle
$$

for all $x, y \in H_{1}$.

Lemma 2.5. ([26]) If $h: H \rightarrow R$ has an L-Lipschitz continuous gradient $\nabla h$, then $\nabla h$ is $\frac{1}{L}$-ism.

We know that a linear bounded operator $A: H \rightarrow H$ is said to be strongly positive if and only if there exists $\bar{\gamma}>0$ such that $\langle A x, x\rangle \geq \bar{\gamma}\|x\|^{2}$ for all $x \in H$. We call such $A$ a strongly positive operator with coefficient $\bar{\gamma}$.

Lemma 2.6. ([27]) Let $H$ be a Hilbert space and let $A$ be a strongly positive bounded linear operator on $H$ with coefficient $\bar{\gamma}>0$. If $0<\delta \leq\|A\|^{-1}$, then $\|I-\delta A\| \leq 1-\delta \bar{\gamma}$. 
Lemma 2.7. ([28]) Let $H$ be a Hilbert space. Let $f: H \rightarrow H$ be a $\rho$-Lipschitzian mapping and let $A: H \rightarrow H$ be a strongly positive bounded linear operator with coefficient $\delta>0$. If $\mu \delta>\eta \rho$, then

$$
\langle(\mu A-\eta f) x-(\mu A-\eta f) y, x-y\rangle \geq(\mu \delta-\eta \rho)\|x-y\|^{2}, \quad x, y \in H,
$$

that is, $\mu A-\eta f$ is strongly monotone with coefficient $\mu \delta-\eta \rho$.

Lemma 2.8. ([29]) The following inequality holds in a Hilbert space $H$

$$
\|x+y\|^{2} \leq\|x\|^{2}+2\langle y, x+y\rangle, \quad \forall x, y \in H .
$$

Lemma 2.9. ([31]) Let $\left\{a_{n}\right\}$ be a sequence of non-negative real numbers such that there exists a subsequence $\left\{a_{n_{j}}\right\}$ of $\left\{a_{n}\right\}$ with $a_{n_{j}}<a_{n_{j}+1}$ for all $j \in N$. Then, there exists a nondecreasing sequence $\left\{m_{k}\right\}$ of $N$ such that $\lim _{k \rightarrow \infty} m_{k}=\infty$, and the following properties are satisfied by all (sufficiently large) number $k \in N$ :

$$
a_{m_{k}} \leq a_{m_{k}+1} \text { and } a_{k} \leq a_{m_{k}+1} .
$$

Indeed, $m_{k}$ is the largest number $n$ in the set $\{1,2, \cdots, k\}$ such that $a_{n}<a_{n+1}$.

Lemma 2.10. ([32]) Assume that $\left\{a_{n}\right\}$ is a sequence of nonnegative real numbers such that

$$
a_{n+1} \leq\left(1-b_{n}\right) a_{n}+c_{n},
$$

where $b_{n}$ is a sequence in $(0,1)$ and $\left\{c_{n}\right\}$ is a sequence such that

(i) $\sum_{n=1}^{\infty} b_{n}=\infty$;

(ii) $\limsup \sup _{n \rightarrow \infty} \frac{c_{n}}{b_{n}} \leq 0$ or $\sum_{n=1}^{\infty}\left|c_{n}\right|<\infty$.

Then $\lim _{n \rightarrow \infty} a_{n}=0$.

\section{MAin RESUlts}

Lemma 3.1. Let $H$ be a Hilbert space. Let $f: H \rightarrow H$ be a $\rho$-Lipschitzian mapping and let $B: H \rightarrow H$ be a strongly positive bounded linear operator with coefficient $\delta>0$. Let $C$ be nonempty, closed and convex subsets of $H$. If $\eta \delta>\gamma \rho$, then the following variational inequality

$$
\langle(\eta B-\gamma f) \hat{x}, \hat{x}-x\rangle \leq 0, \quad \forall x \in C .
$$

has a unique solution. Equivalently, $\hat{x}=P_{C}(I-\eta B+\gamma f) \hat{x}$.

Proof. We show it by contradiction. Suppose that $\hat{x} \in C$ and $\tilde{x} \in C$ are two solution of (3.1) with $\hat{x} \neq \tilde{x}$. Then

$$
\langle(\eta B-\gamma f) \hat{x}, \hat{x}-\tilde{x}\rangle \leq 0
$$

and

$$
\langle(\eta B-\gamma f) \tilde{x}, \tilde{x}-\hat{x}\rangle \leq 0 .
$$

It follows that

$$
\langle(\eta B-\gamma f) \hat{x}-(\eta B-\gamma f) \tilde{x}, \hat{x}-\tilde{x}\rangle \leq 0 .
$$

From $\eta \delta>\gamma \rho$ and Lemma 2.7, we obtain

$$
\langle(\eta B-\gamma f) \hat{x}-(\eta B-\gamma f) \tilde{x}, \hat{x}-\tilde{x}\rangle \geq(\eta \delta-\gamma \rho)\|\hat{x}-\tilde{x}\|^{2} \geq 0 .
$$


This leads to a contradiction. Hence, variational inequality (3.1) has a unique solution and denote it by $\hat{x} \in C$. Since

$$
\langle(\eta B-\gamma f) \hat{x}, \hat{x}-x\rangle \leq 0 \Leftrightarrow\langle\hat{x}-(I-\eta B+\gamma f) \hat{x}, \hat{x}-x\rangle \leq 0, \quad \forall x \in C,
$$

we can obtain from Lemma 2.1 that $\hat{x}=P_{C}(I-\eta B+\gamma f) \hat{x}$.

Theorem 3.1. Let $H_{1}$ and $H_{2}$ be two real Hilbert space. Let $C_{i}$ and $Q_{i}$ be nonempty, closed and convex subsets of $H_{1}$ and $H_{2}$, respectively, for each $1 \leq i \leq N$. Let $A: H_{1} \rightarrow H_{2}$ be a bounded linear operator and let $A^{*}: H_{2} \rightarrow H_{1}$ be the adjoint of $A$. Suppose that MSSFP (1.1) has a nonempty solution set $\Gamma$. Let $f: H_{1} \rightarrow H_{1}$ be a Lipschitzian mapping with coefficient $\rho \geq 0$. Let $B: H_{1} \rightarrow H_{1}$ be a strongly positive bounded linear operator with coefficient $\delta>0$. Let $\left\{x_{n}\right\}$ be a sequence defined as follows:

$$
\left\{\begin{array}{l}
x_{1} \in H_{1} \\
y_{n}=\sum_{i=1}^{N} \mu_{n, i} P_{C_{i}}\left(x_{n}-\xi_{i} A^{*}\left(I-P_{Q_{i}}\right) A x_{n}\right) \\
x_{n+1}=\alpha_{n} \gamma f\left(x_{n}\right)+\left(I-\alpha_{n} \eta B\right) y_{n}
\end{array}\right.
$$

Assume that the following conditions are satisfied:

(i) $\eta \delta>\gamma \rho$;

(ii) $0<\xi_{i}<\frac{2}{\|A\|^{2}}$, $\forall i \in\{1, \cdots, N\}$;

(iii) $\left\{\alpha_{n}\right\} \subset(0,1), \lim _{n \rightarrow \infty} \alpha_{n}=0, \sum_{n=1}^{\infty} \alpha_{n}=\infty$;

(iv) $\sum_{i=1}^{N} \mu_{n, i}=1, \sum_{n=0}^{\infty} \sum_{i=1}^{N}\left|\mu_{n, i}-\mu_{(n-1), i}\right|<\infty$ and $\mu_{n, i}>0$, for $i \in\{1, \cdots, N\}$.

Then $\left\{x_{n}\right\}$ converges strongly to a point $x^{*} \in \Gamma$, which is the unique solution of the following variational inequality

$$
\left\langle(\eta B-\gamma f) x^{*}, x^{*}-x\right\rangle \leq 0, \forall x \in \Gamma
$$

Equivalently, $x^{*}=P_{\Gamma}(I-\eta B+\gamma f) x^{*}$.

Proof. The proof is split into five steps.

Step 1. We show that $\left\{x_{n}\right\}$ is bounded.

Let $y_{n}=\sum_{i=1}^{N} \mu_{n, i} S_{i} x_{n}=T_{n} x_{n}$, where

$$
S_{i}=P_{C_{i}}\left(I-\xi_{i} A^{*}\left(I-P_{Q_{i}}\right) A\right)
$$

For nay $p \in \Gamma$, we have $S_{i} p=p, \forall i \in\{1, \cdots, N\}$ and $T_{n} p=p$. Since $P_{Q_{i}}$ is firmly nonexpansive, we have from Lemma 2.4 that $A^{*}\left(I-P_{Q_{i}}\right) A$ is $\frac{1}{\|A\|^{2}}$-ism. From $0<\xi_{i}<\frac{2}{\|A\|^{2}}$, we have that $I-\xi_{i} A^{\star}\left(I-P_{Q_{i}}\right) A$ is nonexpansive. Then $S_{i}$ is nonexpansive. Obviously, $T_{n}$ is also nonexpansive. Then

$$
\left\|y_{n}-p\right\|=\left\|T_{n} x_{n}-T_{n} p\right\| \leq\left\|x_{n}-p\right\| .
$$


From the condition $\lim _{n \rightarrow \infty} \alpha_{n}=0$, we may assume that, without loss of generality, $\alpha_{n}<\frac{1}{\eta\|B\|}$ for all $n$. It follows from Lemma 2.6 that

$$
\begin{aligned}
\left\|x_{n+1}-p\right\| & =\left\|\alpha_{n} \gamma f\left(x_{n}\right)+\left(I-\alpha_{n} \eta B\right) y_{n}-p\right\| \\
& =\left\|\alpha_{n}\left(\gamma f\left(x_{n}\right)-\eta B p\right)+\left(I-\alpha_{n} \eta B\right)\left(y_{n}-p\right)\right\| \\
& \leq \alpha_{n}\left\|\gamma f\left(x_{n}\right)-\eta B p\right\|+\left\|I-\alpha_{n} \eta B\right\|\left\|y_{n}-p\right\| \\
& =\alpha_{n}\left\|\gamma f\left(x_{n}\right)-\gamma f(p)+\gamma f(p)-\eta B p\right\|+\left\|1-\alpha_{n} \eta B\right\|\left\|y_{n}-p\right\| \\
& \leq \alpha_{n} \gamma \rho\left\|x_{n}-p\right\|+\alpha_{n}\|\gamma f(p)-\eta B p\|+\left(1-\alpha_{n} \eta \delta\right)\left\|x_{n}-p\right\| \\
& =\left[1-\alpha_{n}(\eta \delta-\gamma \rho)\right]\left\|x_{n}-p\right\|+\alpha_{n}\|\gamma f(p)-\eta B p\| \\
& =\left[1-\alpha_{n}(\eta \delta-\gamma \rho)\right]\left\|x_{n}-p\right\|+\alpha_{n}(\eta \delta-\gamma \rho) \frac{\|\gamma f(p)-\eta B p\|}{\eta \delta-\gamma \rho} \\
& \leq \max \left\{\left\|x_{n}-p\right\|, \frac{\|\gamma f(p)-\eta B p\|}{\eta \delta-\gamma \rho}\right\} \\
& \leq \cdots \\
& \leq \max \left\{\left\|x_{0}-p\right\|, \frac{\|\gamma f(p)-\eta B p\|}{\eta \delta-\gamma \rho}\right\} .
\end{aligned}
$$

Therefore, $\left\{x_{n}\right\}$ is bounded. $\left\{y_{n}\right\},\left\{f\left(x_{n}\right)\right\},\left\{S_{i} x_{n}\right\},\left\{T_{n} x_{n}\right\}$ and $\left\{B T_{n} x_{n}\right\}$ are also bounded.

Step 2. We show that $\left\|x_{n}-T_{n} x_{n}\right\| \rightarrow 0$ as $n \rightarrow \infty$.

Observe that

$$
\left\|x_{n+1}-T_{n} x_{n}\right\|=\alpha_{n}\left\|\gamma f\left(x_{n}\right)-\eta B T_{n} x_{n}\right\| \rightarrow 0(\text { as } n \rightarrow \infty) .
$$

So, we only need to prove $\left\|x_{n+1}-x_{n}\right\| \rightarrow 0$. Indeed,

$$
\begin{aligned}
\left\|x_{n+1}-x_{n}\right\|= & \left\|\alpha_{n} \gamma f\left(x_{n}\right)+\left(I-\alpha_{n} \eta B\right) y_{n}-\alpha_{n-1} \gamma f\left(x_{n-1}\right)-\left(I-\alpha_{n-1} \eta B\right) y_{n-1}\right\| \\
= & \| \alpha_{n} \gamma f\left(x_{n}\right)-\alpha_{n} \gamma f\left(x_{n-1}\right)+\alpha_{n} \gamma f\left(x_{n-1}\right)-\alpha_{n-1} \gamma f\left(x_{n-1}\right)+\left(I-\alpha_{n} \eta B\right) T_{n} x_{n} \\
& -\left(I-\alpha_{n} \eta B\right) T_{n-1} x_{n-1}+\left(I-\alpha_{n} \eta B\right) T_{n-1} x_{n-1}-\left(I-\alpha_{n-1} \eta B\right) T_{n-1} x_{n-1} \| \\
\leq & \alpha_{n} \gamma\left\|f\left(x_{n}\right)-f\left(x_{n-1}\right)\right\|+\left|\alpha_{n}-\alpha_{n-1}\right|\left\|\gamma f\left(x_{n-1}\right)\right\| \\
& +\left\|I-\alpha_{n} \eta B\right\|\left\|T_{n} x_{n}-T_{n-1} x_{n-1}\right\|+\left|\alpha_{n}-\alpha_{n-1}\right|\left\|\eta B T_{n-1} x_{n-1}\right\| \\
\leq & \alpha_{n} \gamma \rho\left\|x_{n}-x_{n-1}\right\|+\left|\alpha_{n}-\alpha_{n-1}\right|\left\|\gamma f\left(x_{n-1}\right)\right\| \\
& +\left(1-\alpha_{n} \eta \delta\right)\left\|T_{n} x_{n}-T_{n-1} x_{n-1}\right\|+\left|\alpha_{n}-\alpha_{n-1}\right|\left\|\eta B T_{n-1} x_{n-1}\right\| \\
\leq & \alpha_{n} \gamma \rho\left\|x_{n}-x_{n-1}\right\|+\left|\alpha_{n}-\alpha_{n-1}\right|\left\|\gamma f\left(x_{n-1}\right)\right\| \\
& +\left(1-\alpha_{n} \eta \delta\right)\left(\left\|T_{n} x_{n}-T_{n} x_{n-1}\right\|+\left\|T_{n} x_{n-1}-T_{n-1} x_{n-1}\right\|\right) \\
& +\left|\alpha_{n}-\alpha_{n-1}\right|\left\|\eta B T_{n-1} x_{n-1}\right\| \\
\leq & \alpha_{n} \gamma \rho\left\|x_{n}-x_{n-1}\right\|+\left|\alpha_{n}-\alpha_{n-1}\right|\left\|\gamma f\left(x_{n-1}\right)\right\| \\
& +\left(1-\alpha_{n} \eta \delta\right)\left(\left\|x_{n}-x_{n-1}\right\|+\left\|\sum_{i=1}^{N} \mu_{n, i} S_{i} x_{n-1}-\sum_{i=1}^{N} \mu_{(n-1), i} S_{i} x_{n-1}\right\|\right) \\
& +\left|\alpha_{n}-\alpha_{n-1}\right|\left\|\eta B T_{n-1} x_{n-1}\right\|
\end{aligned}
$$




$$
\begin{aligned}
\leq & \alpha_{n} \gamma \rho\left\|x_{n}-x_{n-1}\right\|+\left|\alpha_{n}-\alpha_{n-1}\right|\left\|\gamma f\left(x_{n-1}\right)\right\| \\
& +\left(1-\alpha_{n} \eta \delta\right)\left(\left\|x_{n}-x_{n-1}\right\|+\sum_{i=1}^{N}\left|\mu_{n, i}-\mu_{(n-1), i}\right|\left\|S_{i} x_{n-1}\right\|\right) \\
& +\left|\alpha_{n}-\alpha_{n-1}\right|\left\|\eta B T_{n-1} x_{n-1}\right\| \\
= & {\left[1-\alpha_{n}(\eta \delta-\gamma \rho)\right]\left\|x_{n}-x_{n-1}\right\|+\left|\alpha_{n}-\alpha_{n-1}\right|\left(\left\|\gamma f\left(x_{n-1}\right)\right\|+\left\|\eta B T_{n-1} x_{n-1}\right\|\right) } \\
& +\left(1-\alpha_{n} \eta \delta\right) \sum_{i=1}^{N}\left|\mu_{n, i}-\mu_{(n-1), i}\right|\left\|S_{i} x_{n-1}\right\| \\
\leq & {\left[1-\alpha_{n}(\eta \delta-\gamma \rho)\right]\left\|x_{n}-x_{n-1}\right\|+\left|\alpha_{n}-\alpha_{n-1}\right|\left(\left\|\gamma f\left(x_{n-1}\right)\right\|+\left\|\eta B T_{n-1} x_{n-1}\right\|\right) } \\
& +\sum_{i=1}^{N}\left|\mu_{n, i}-\mu_{(n-1), i}\right|\left\|S_{i} x_{n-1}\right\| .
\end{aligned}
$$

Let

$$
M_{1}=\sup _{n, i}\left\{\left\|\gamma f\left(x_{n-1}\right)\right\|+\left\|\eta B T_{n-1} x_{n-1}\right\|,\left\|S_{i} x_{n-1}\right\|\right\}
$$

It follows that

$$
\left\|x_{n+1}-x_{n}\right\| \leq\left[1-\alpha_{n}(\eta \delta-\gamma \rho)\right]\left\|x_{n}-x_{n-1}\right\|+\left(\left|\alpha_{n}-\alpha_{n-1}\right|+\sum_{i=1}^{N}\left|\mu_{n, i}-\mu_{(n-1), i}\right|\right) M_{1} .
$$

From the conditions $\sum_{n=0}^{\infty} \alpha_{n}=\infty, \sum_{n=0}^{\infty}\left|\alpha_{n}-\alpha_{n-1}\right|<\infty$ and $\sum_{n=0}^{\infty} \sum_{i=1}^{N}\left|\mu_{n, i}-\mu_{(n-1), i}\right|<\infty$ and Lemma 2.10, we obtain

$$
\left\|x_{n+1}-x_{n}\right\| \rightarrow 0 \quad(\text { as } n \rightarrow \infty) .
$$

Therefore, $\left\|x_{n}-T_{n} x_{n}\right\| \rightarrow 0$, as $n \rightarrow \infty$.

Step 3. We show that $\omega_{\omega}\left(x_{n}\right) \subseteq \Gamma$.

To see this, we take $q \in \omega_{\omega}\left(x_{n}\right)$ and assume that $x_{n_{l}} \rightarrow q$ as $l \rightarrow \infty$ for some subsequence $\left\{x_{n_{l}}\right\}$ of $\left\{x_{n}\right\}$. We know that $T_{n}=\sum_{i=1}^{N} \mu_{n, i} S_{i}$. From the conditions $\mu_{n, i}>0$ and $\sum_{i=1}^{N} \mu_{n, i}=1$, for $\forall i \in\{1, \cdots, N\}$, we may assume, with no loss of generality, that

$$
\mu_{n_{l}, i} \rightarrow \mu_{i}(\text { as } l \rightarrow \infty), \forall 1 \leq i \leq N .
$$

It is obvious that each $\mu_{i}>0$ and $\sum_{i=1}^{N} \mu_{i}=1$. And we also have

$$
T_{n_{l}} x \rightarrow T x, \quad \forall x \in H_{1}, \quad(\text { as } l \rightarrow \infty),
$$

where

$$
T=\sum_{i=1}^{N} \mu_{i} S_{i}
$$

By using Lemma 2.3, we have that $T$ is nonexpansive and $F(T)=\bigcap_{i=1}^{N} F\left(S_{i}\right)=\Gamma$. It follows that

$$
\begin{aligned}
\left\|x_{n_{l}}-T x_{n_{l}}\right\| & \leq\left\|x_{n_{l}}-T_{n_{l}} x_{n_{l}}\right\|+\left\|T_{n_{l}} x_{n_{l}}-T x_{n_{l}}\right\| \\
& \leq\left\|x_{n_{l}}-T_{n_{l}} x_{n_{l}}\right\|+\sum_{i=1}^{N}\left|\mu_{n_{l}, i}-\mu_{i}\right|\left\|S_{i} x_{n_{l}}\right\| \\
& \rightarrow 0(l \rightarrow \infty) .
\end{aligned}
$$

It follows from Lemma 2.3 that $q \in F(T)=\Gamma$, that is, $\omega_{\omega}\left(x_{n}\right) \subseteq \Gamma$. 
Step 4. We show that

$$
\limsup _{n \rightarrow \infty}\left\langle(\eta B-\gamma f) x^{*}, x^{*}-x_{n}\right\rangle \leq 0 .
$$

where $x^{*}$ is the unique solution of variational inequality (3.1).

Indeed, take a subsequence $\left\{x_{n_{j}}\right\}$ of $\left\{x_{n}\right\}$ such that

$$
\limsup _{n \rightarrow \infty}\left\langle(\eta B-\gamma f) x^{*}, x^{*}-x_{n}\right\rangle=\lim _{j \rightarrow \infty}\left\langle(\eta B-\gamma f) x^{*}, x^{*}-x_{n_{j}}\right\rangle .
$$

Since $\left\{x_{n}\right\}$ is bounded, without loss of generality, we may assume that $x_{n_{j}} \rightarrow \bar{x} \in \Gamma$. Then

$$
\limsup _{n \rightarrow \infty}\left\langle(\eta B-\gamma f) x^{*}, x^{*}-x_{n}\right\rangle=\left\langle(\eta B-\gamma f) x^{*}, x^{*}-\bar{x}\right\rangle \leq 0 .
$$

Step 5. We show that $x_{n} \rightarrow x^{*}(n \rightarrow \infty)$.

From Lemma 2.6 and Lemma 2.8, we have

$$
\begin{aligned}
\left\|x_{n+1}-x^{*}\right\|^{2}= & \left\|\alpha_{n} \gamma f\left(x_{n}\right)+\left(I-\alpha_{n} \eta B\right) y_{n}-x^{*}\right\|^{2} \\
= & \left\|\alpha_{n}\left(\gamma f\left(x_{n}\right)-\eta B x^{*}\right)+\left(I-\alpha_{n} \eta B\right)\left(y_{n}-x^{*}\right)\right\|^{2} \\
\leq & \left\|\left(I-\alpha_{n} \eta B\right)\left(y_{n}-x^{*}\right)\right\|^{2}+2 \alpha_{n}\left\langle\gamma f\left(x_{n}\right)-\eta B x^{*}, x_{n+1}-x^{*}\right\rangle \\
\leq & \left(1-\alpha_{n} \eta \delta\right)^{2}\left\|y_{n}-x^{*}\right\|^{2}+2 \alpha_{n}\left\langle\gamma f\left(x_{n}\right)-\eta B x^{*}, x_{n+1}-x^{*}\right\rangle \\
= & \left(1-\alpha_{n} \eta \delta\right)^{2}\left\|y_{n}-x^{*}\right\|^{2}+2 \alpha_{n}\left\langle\gamma f\left(x_{n}\right)-\gamma f\left(x^{*}\right), x_{n+1}-x^{*}\right\rangle \\
& +2 \alpha_{n}\left\langle\gamma f\left(x^{*}\right)-\eta B x^{*}, x_{n+1}-x^{*}\right\rangle \\
\leq & \left(1-\alpha_{n} \eta \delta\right)^{2}\left\|y_{n}-x^{*}\right\|^{2}+2 \alpha_{n} \gamma \rho\left\|x_{n}-x^{*}\right\|\left\|x_{n+1}-x^{*}\right\| \\
& +2 \alpha_{n}\left\langle\gamma f\left(x^{*}\right)-\eta B x^{*}, x_{n+1}-x^{*}\right\rangle \\
\leq & \left(1-\alpha_{n} \eta \delta\right)^{2}\left\|x_{n}-x^{*}\right\|^{2}+\alpha_{n} \gamma \rho\left(\left\|x_{n}-x^{*}\right\|^{2}+\left\|x_{n+1}-x^{*}\right\|^{2}\right) \\
& +2 \alpha_{n}\left\langle\gamma f\left(x^{*}\right)-\eta B x^{*}, x_{n+1}-x^{*}\right\rangle .
\end{aligned}
$$

Thus

$$
\left(1-\alpha_{n} \gamma \rho\right)\left\|x_{n+1}-x^{*}\right\|^{2} \leq\left[\left(1-\alpha_{n} \eta \delta\right)^{2}+\alpha_{n} \gamma \rho\right]\left\|x_{n}-x^{*}\right\|^{2}+2 \alpha_{n}\left\langle\gamma f\left(x^{*}\right)-\eta B x^{*}, x_{n+1}-x^{*}\right\rangle .
$$

Since $\eta \delta>\gamma \rho$ and

$$
0<\alpha_{n} \leq \frac{1}{\eta\|B\|} \leq \frac{1}{\eta \delta}
$$

we have $1-\alpha_{n} \gamma \rho>1-\alpha_{n} \eta \delta \geq 0$. Hence

$$
\begin{aligned}
\left\|x_{n+1}-x^{*}\right\|^{2} \leq & \frac{\left(1-\alpha_{n} \eta \delta\right)^{2}+\alpha_{n} \gamma \rho}{1-\alpha_{n} \gamma \rho}\left\|x_{n}-x^{*}\right\|^{2}+\frac{2 \alpha_{n}}{1-\alpha_{n} \gamma \rho}\left\langle\gamma f\left(x^{*}\right)-\eta B x^{*}, x_{n+1}-x^{*}\right\rangle \\
= & {\left[1-\frac{2 \alpha_{n}(\eta \delta-\gamma \rho)}{1-\alpha_{n} \gamma \rho}\right]\left\|x_{n}-x^{*}\right\|^{2}+\frac{2 \alpha_{n}}{1-\alpha_{n} \gamma \rho}\left\langle\gamma f\left(x^{*}\right)-\eta B x^{*}, x_{n+1}-x^{*}\right\rangle } \\
& +\frac{\alpha_{n}^{2} \eta^{2} \delta^{2}}{1-\alpha_{n} \gamma \rho}\left\|x_{n}-x^{*}\right\|^{2} \\
\leq & {\left[1-\frac{2 \alpha_{n}(\eta \delta-\gamma \rho)}{1-\alpha_{n} \gamma \rho}\right]\left\|x_{n}-x^{*}\right\|^{2} } \\
& +\frac{2 \alpha_{n}(\eta \delta-\gamma \rho)}{1-\alpha_{n} \gamma \rho}\left(\frac{\left\langle\gamma f\left(x^{*}\right)-\eta B x^{*}, x_{n+1}-x^{*}\right\rangle}{\eta \delta-\gamma \rho}+\alpha_{n} M_{2}\right) .
\end{aligned}
$$


where $M_{2}$ is a constant satisfying

$$
M_{2}=\sup _{n \geq 0}\left\{\frac{\eta^{2} \delta^{2}}{2(\eta \delta-\gamma \rho)}\left\|x_{n}-x^{*}\right\|^{2}\right\} .
$$

From the condition $\sum_{n=0}^{\infty} \alpha_{n}=\infty, \lim _{n \rightarrow \infty} \alpha_{n}=0$ and (3.5), we have

$$
\sum_{n=0}^{\infty} \frac{2 \alpha_{n}(\eta \delta-\gamma \rho)}{1-\alpha_{n} \gamma \rho}>\sum_{n=0}^{\infty} 2 \alpha_{n}(\eta \delta-\gamma \rho)=\infty
$$

and

$$
\limsup _{n \rightarrow \infty}\left(\frac{\left\langle\gamma f\left(x^{*}\right)-\eta B x^{*}, x_{n+1}-x^{*}\right\rangle}{\eta \delta-\gamma \rho}+\alpha_{n} M_{2}\right) \leq 0 .
$$

From Lemma 2.10, we can obtain that $\left\|x_{n}-x^{*}\right\| \rightarrow 0$ as $n \rightarrow \infty$.

Next, we give the other strong convergence theorem in Hilbert spaces.

Theorem 3.2. Let $H_{1}$ and $H_{2}$ be two real Hilbert space. Let $C_{i}$ and $Q_{i}$ be nonempty, closed and convex subsets of $H_{1}$ and $H_{2}$, respectively, for each $1 \leq i \leq N$. Let $A: H_{1} \rightarrow H_{2}$ be a bounded linear operator and $A^{*}: H_{2} \rightarrow H_{1}$ be the adjoint of $A$. Suppose that MSSFP (1.1) has a nonempty solution set $\Gamma$. Let $f: H_{1} \rightarrow H_{1}$ be a Lipschitzian mapping with coefficient $\rho \geq 0$. Let $B: H_{1} \rightarrow H_{1}$ be a strongly positive bounded linear operator with coefficient $\delta>0$. Let $\left\{x_{n}\right\}$ be a sequence defined as follows

$$
\left\{\begin{array}{l}
x_{1} \in H_{1} \\
y_{n}=P_{C_{[n]}}\left(x_{n}-\xi_{[n]} A^{*}\left(I-P_{Q_{[n]}}\right) A x_{n}\right), \\
x_{n+1}=\alpha_{n} \gamma f\left(x_{n}\right)+\left(I-\alpha_{n} \eta B\right) y_{n} .
\end{array}\right.
$$

where $[n]=n(\bmod N)$ are the mod functions taking values in $\{1, \cdots, N\}$. Assume that the following conditions are satisfied:

(i) $\eta \delta>\gamma \rho$;

(ii) $0<\xi_{i}<\frac{2}{\|A\|^{2}}$, for $\forall i \in\{1, \cdots, N\}$;

(iii) $\left\{\alpha_{n}\right\} \subset(0,1), \lim _{n \rightarrow \infty} \alpha_{n}=0, \sum_{n} \alpha_{n}=\infty$.

Then $\left\{x_{n}\right\}$ converges strongly to a point $x^{*} \in \Gamma$, which solves variational inequality (3.1).

Proof. First, we show that sequence $\left\{x_{n}\right\}$ is bounded. Let $y_{n}=T_{[n]} x_{n}$, where

$$
T_{[n]}=P_{C_{[n]}}\left(I-\xi_{[n]} A^{*}\left(I-P_{Q_{[n]}}\right) A .\right.
$$

Picking any $p \in \Gamma$, we have $T_{[n]} p=p$. Since $P_{Q_{[n]}}$ is firmly nonexpansive, it follows from Lemma 2.4 that $A^{*}\left(I-P_{Q_{[n]}}\right) A$ is $\frac{1}{\|A\|^{2}}$-ism. From $0<\xi_{[n]}<\frac{2}{\|A\|^{2}}$, we have that $I-\xi_{[n]} A^{*}\left(I-P_{Q_{[n]}}\right) A$ is nonexpansive. Then $T_{[n]}$ is also nonexpansive. It follows that

$$
\left\|y_{n}-p\right\|=\left\|T_{[n]} x_{n}-T_{[n]} p\right\| \leq\left\|x_{n}-p\right\| .
$$

Using (3.4), we get that $\left\{x_{n}\right\}$ is bounded. Hence $\left\{y_{n}\right\},\left\{f\left(x_{n}\right)\right\}$ and $\left\{T_{[n]} x_{n}\right\}$ are also bounded.

Next, we show that $\left\|x_{n}-x^{*}\right\| \rightarrow 0$ as $n \rightarrow \infty$, where $x^{*}$ is the unique solution of variational inequality (3.1). Since $A^{*}\left(I-P_{Q_{[n]}}\right) A$ is $\frac{1}{\|A\|^{2}}$-ism, it follows from Proposition 2.1 and Proposition 2.2 that $\xi_{[n]} A^{*}(I-$ $\left.P_{Q_{[n]}}\right) A$ is $\frac{1}{\xi_{[n]}\|A\|^{2}}$-ism, $I-\xi_{[n]} A^{*}\left(I-P_{Q_{[n]}}\right) A$ is $\frac{\xi_{[n]}\|A\|^{2}}{2}$-av and

$$
T_{[n]}=P_{C_{[n]}}\left(I-\xi_{[n]} A^{*}\left(I-P_{Q_{[n]}}\right) A\right.
$$


is $\frac{2+\xi_{[n]}\|A\|^{2}}{4}-$ av. Then

$$
\begin{aligned}
\left\|y_{n}-x^{*}\right\|^{2} & =\left\|T_{[n]} x_{n}-x^{*}\right\|^{2} \\
& \leq\left\|x_{n}-x^{*}\right\|^{2}-\frac{1-\frac{2+\xi_{[n]}\|A\|^{2}}{4}}{\frac{2+\xi_{[n]}\|A\|^{2}}{4}}\left\|T_{[n]} x_{n}-x_{n}\right\|^{2} \\
& =\left\|x_{n}-x^{*}\right\|^{2}-\frac{2-\xi_{[n]}\|A\|^{2}}{2+\xi_{[n]}\|A\|^{2}}\left\|T_{[n]} x_{n}-x_{n}\right\|^{2} .
\end{aligned}
$$

Observe that

$$
\begin{aligned}
\left\|x_{n+1}-x^{*}\right\|^{2}= & \left\|\alpha_{n} \gamma f\left(x_{n}\right)+\left(I-\alpha_{n} \eta B\right) y_{n}-x^{*}\right\|^{2} \\
= & \left\|\alpha_{n}\left(\gamma f\left(x_{n}\right)-\eta B x^{*}\right)+\left(I-\alpha_{n} \eta B\right)\left(y_{n}-x^{*}\right)\right\|^{2} \\
= & \alpha_{n}^{2}\left\|\gamma f\left(x_{n}\right)-\eta B x^{*}\right\|^{2}+\left\|\left(I-\alpha_{n} \eta B\right)\left(y_{n}-x^{*}\right)\right\|^{2} \\
& +2 \alpha_{n}\left\langle\gamma f\left(x_{n}\right)-\eta B x^{*},\left(I-\alpha_{n} \eta B\right)\left(y_{n}-x^{*}\right)\right\rangle \\
\leq & \alpha_{n}^{2}\left\|\gamma f\left(x_{n}\right)-\eta B x^{*}\right\|^{2}+\left(1-\alpha_{n} \eta \delta\right)^{2}\left\|y_{n}-x^{*}\right\|^{2} \\
& +2 \alpha_{n}\left(1-\alpha_{n} \eta \delta\right)\left\|\gamma f\left(x_{n}\right)-\eta B x^{*}\right\|\left\|y_{n}-x^{*}\right\| \\
\leq & \alpha_{n}^{2}\left\|\gamma f\left(x_{n}\right)-\eta B x^{*}\right\|^{2}+\left(1-\alpha_{n} \eta \delta\right)^{2}\left(\left\|x_{n}-x^{*}\right\|^{2}-\frac{2-\xi_{[n]}\|A\|^{2}}{2+\xi_{[n]}\|A\|^{2}}\left\|T_{[n]} x_{n}-x_{n}\right\|^{2}\right) \\
& +2 \alpha_{n}\left(1-\alpha_{n} \eta \delta\right)\left\|\gamma f\left(x_{n}\right)-\eta B x^{*}\right\|\left\|x_{n}-x^{*}\right\| .
\end{aligned}
$$

Then

$$
\begin{aligned}
\left(1-\alpha_{n} \eta \delta\right)^{2} \frac{2-\xi_{[n]}\|A\|^{2}}{2+\xi_{[n]}\|A\|^{2}}\left\|T_{[n]} x_{n}-x_{n}\right\|^{2} \leq & \left(1-\alpha_{n} \eta \delta\right)^{2}\left\|x_{n}-x^{*}\right\|^{2}-\left\|x_{n+1}-x^{*}\right\|^{2} \\
& +\alpha_{n}^{2}\left\|\gamma f\left(x_{n}\right)-\eta B x^{*}\right\|^{2}+2 \alpha_{n}\left(1-\alpha_{n} \eta \delta\right) \\
& \times\left\|\gamma f\left(x_{n}\right)-\eta B x^{*}\right\|\left\|\left(x_{n}-x^{*}\right)\right\| \\
\leq & \left\|x_{n}-x^{*}\right\|^{2}-\left\|x_{n+1}-x^{*}\right\|^{2}+\alpha_{n}^{2} \eta^{2} \delta^{2}\left\|x_{n}-x^{*}\right\|^{2} \\
& +\alpha_{n}^{2}\left\|\gamma f\left(x_{n}\right)-\eta B x^{*}\right\|^{2}+2 \alpha_{n}\left(1-\alpha_{n} \eta \delta\right) \\
& \times\left\|\gamma f\left(x_{n}\right)-\eta B x^{*}\right\|\left\|x_{n}-x^{*}\right\| .
\end{aligned}
$$

Next, we analyze inequality (3.7) by considering the following two cases.

Case 1. Assume that there exists $n_{0}$ large enough such that $\left\|x_{n+1}-x^{*}\right\|^{2} \leq\left\|x_{n}-x^{*}\right\|^{2}$ for all $n \geq n_{0}$. Since $\left\|x_{n}-x^{*}\right\|^{2}$ is bounded, we have that $\lim _{n \rightarrow \infty}\left\|x_{n}-x^{*}\right\|^{2}$ exists. Since $\lim _{n \rightarrow \infty} \alpha_{n}=0,0<\xi_{[n]}<\frac{2}{\|A\|^{2}}$ for $\forall n \geq 1,\left\{x_{n}\right\}$ and $\left\{f\left(x_{n}\right)\right\}$ are bounded, we can obtain

$$
\left\|T_{[n]} x_{n}-x_{n}\right\| \rightarrow 0(n \rightarrow \infty)
$$

Since

$$
\left\|x_{n+1}-T_{[n]} x_{n}\right\|=\left\|x_{n+1}-y_{n}\right\|=\alpha_{n}\left\|\gamma f\left(x_{n}\right)-\eta B y_{n}\right\| \rightarrow 0(n \rightarrow \infty)
$$

we have

$$
\left\|x_{n+1}-x_{n}\right\| \rightarrow 0(n \rightarrow \infty) .
$$

Next, we show that $\omega_{\omega}\left(x_{n}\right) \subseteq \Gamma$. To see this, we take $q \in \omega_{\omega}\left(x_{n}\right)$ and assume that $x_{n_{l}} \rightarrow q$ as $l \rightarrow \infty$ for some subsequence $\left\{x_{n_{l}}\right\}$ of $\left\{x_{n}\right\}$. We may further assume $n_{l}=k(\bmod N)$ for all $l$. From $(3.8)$, we have 
$x_{n_{l}+j} \rightarrow q$ for all $j \geq 0$. Then

$$
\left\|x_{n_{l}+j}-T_{[k+j]} x_{n_{l}+j}\right\|=\left\|x_{n_{l}+j}-T_{\left[n_{l}+j\right]} x_{n_{l}+j}\right\| \rightarrow 0 \quad(\text { as } l \rightarrow \infty) .
$$

By Lemma 2.2, we can obtain $q \in F\left(T_{[k+j]}\right)$ for all $j$. Hence, $q \in \Gamma$, that is, $\omega_{\omega}\left(x_{n}\right) \subseteq \Gamma$. The remaining of the proof is the same as that of Theorem 3.1, we omit it. Therefore, we can obtain that $x_{n} \rightarrow x^{*}(n \rightarrow \infty)$.

Case 2. Assume that there exists a subsequence $\left\{\left\|x_{n_{j}}-x^{*}\right\|^{2}\right\}$ of $\left\{\left\|x_{n}-x^{*}\right\|^{2}\right\}$ such that $\left\|x_{n_{j}}-x^{*}\right\|^{2}<$ $\left\|x_{n_{j}+1}-x^{*}\right\|^{2}$ for all $j \in N$. It follows from Lemma 2.9 that there exists a nondecreasing sequence $\left\{m_{k}\right\}$ of $N$ such that $\lim _{k \rightarrow \infty} m_{k}=\infty$, and the following inequalities hold for all $k \in N$ :

$$
\left\|x_{m_{k}}-x^{*}\right\|^{2} \leq\left\|x_{m_{k}+1}-x^{*}\right\|^{2} \text { and }\left\|x_{k}-x^{*}\right\|^{2} \leq\left\|x_{m_{k}+1}-x^{*}\right\|^{2} .
$$

Similarly, we can get

$$
\left\|T_{\left[m_{k}\right]} x_{m_{k}}-x_{m_{k}}\right\| \rightarrow 0(n \rightarrow \infty) .
$$

Following an argument similar to that in Case 1 , we have $\omega_{\omega}\left(x_{m_{k}}\right) \subseteq \Gamma$. Also, we have

$$
\limsup _{n \rightarrow \infty}\left\langle(\eta B-\gamma f) x^{*}, x^{*}-x_{m_{k}}\right\rangle \leq 0
$$

and

$$
\begin{aligned}
\left\|x_{m_{k}+1}-x^{*}\right\|^{2} \leq & {\left[1-\frac{2 \alpha_{m_{k}}(\eta \delta-\gamma \rho)}{1-\alpha_{m_{k}} \gamma \rho}\right]\left\|x_{m_{k}}-x^{*}\right\|^{2} } \\
& +\frac{2 \alpha_{m_{k}}(\eta \delta-\gamma \rho)}{1-\alpha_{m_{k}} \gamma \rho}\left(\frac{\left\langle\gamma f\left(x^{*}\right)-\eta B x^{*}, x_{m_{k}+1}-x^{*}\right\rangle}{\eta \delta-\gamma \rho}+\alpha_{m_{k}} M_{2}\right) .
\end{aligned}
$$

where $M$ is a constant satisfying

$$
M_{2}=\sup _{k \geq 0}\left\{\frac{\eta^{2} \delta^{2}}{2(\eta \delta-\gamma \rho)}\left\|x_{m_{k}}-x^{*}\right\|^{2}\right\} .
$$

By the same argument as in Case 1, we obtain that $\left\|x_{m_{k}}-x^{*}\right\| \rightarrow 0$ as $k \rightarrow \infty$. Using (3.9), we get $\left\|x_{k}-x^{*}\right\| \leq\left\|x_{m_{k}}-x^{*}\right\|, \forall k \in N$. Therefore, $x_{k} \rightarrow x^{*}$ as $k \rightarrow \infty$. This ends the proof.

\section{Acknowledgments}

The authors are grateful to the anonymous referees for useful suggestions which improved the contents of this paper. The second author was supported by the Natural Science Foundation of Shandong Province of China (ZR2017LA001) and Youth Foundation of Linyi University (LYDX2016BS023).

\section{REFERENCES}

[1] Y. Censor, T. Elfving, A multiprojection algorithm using Bregman projections in a product space, Numer. Algor. 8 (1994), 221-239.

[2] C. Byrne, Iterative oblique projection onto convex sets and the split feasibility problem, Inverse Problems, 18 (2002), 441-453.

[3] C. Byrne, A unified treatment of some iterative algorithms in signal processing and image reconstruction, Inverse Probl. 20 (2004), 103-120.

[4] X. Qin, A. Petrusel, J.C. Yao, CQ iterative algorithms for fixed points of nonexpansive mappings and split feasibility problems in Hilbert spaces, J. Nonlinear Convex Anal. 19 (2018), 157-165.

[5] L.C. Ceng, Q.H. Ansari, J.C. Yao, An extragradient method for solving split feasibility and fixed point problems, Comput. Math. Appl. 64 (2012), 633-642.

[6] L.C. Ceng, Q.H. Ansari, J.C. Yao Relaxed extragradient methods for finding minimum-norm solutions of the split feasibility problem, Nonlinear Anal. 75 (2012), 2116-2125. 
[7] S.Y. Cho, X. Qin, J.C. Yao, Y. Yao, Viscosity approrimation splitting methods for monotone and nonexpansive operators in Hilbert spaces, J. Nonlinear Convex Anal. 19 (2018), 251-264.

[8] J. Zhao, Solving split equality fixed-point problem of quasi-nonexpansive mappings without prior knowledge of operators norms, Optimization 64 (2015), 2619-2630.

[9] L. Liu, A hybrid steepest descent method for solving split feasibility problems involving nonexpansive mappings, J Nonlinear Convex Anal. 20 (2019), 471-488.

[10] X. Qin, J.C. Yao, A viscosity iterative method for a split feasibility problem, J. Nonlinear Convex Anal. (2019), in press.

[11] H. K. Xu, A variable Krasnosel'skii-Mann algorithm and the multiple-set split feasibility problem, Inverse Probl. 22 (2006), 2021-2034.

[12] H. K. Xu, Iterative methods for split feasibility problem in infinite-dimensional Hilbert spaces, Inverse Problems, 26 (2010), (105018).

[13] K. Sitthithakerngkiet, J. Deepho, P. Kumam, Modified hybrid steepest method for the split feasibility problem in image recovery of inverse problems, Numer. Funct. Anal. Optim. 38 (2017), 507-522.

[14] Y. Censor, T. Elfving, N. Kopf, T. Bortfeld, The multiple-sets split feasibility problem and its applications for inverse problems, Inverse Probl. 21 (2005) 2071-2084.

[15] Q. Dong, S. He, Two projection algorithms for the multiple-sets split feasibility problem, J. Appl. Math. 2013 (2013), Article ID 347401.

[16] Z. Li, D. Han, W. Zhang, A self-adaptive projection-type method for nonlinear multiple-sets split feasibility problem, Inverse Probl. Sci. Eng. 21 (2013), 155-170.

[17] W. Zhang, D. Han, X. Yuan, An efficient simultaneous method for the constrained multiple-sets split feasibility problem, Comput. Optim. Appl. 52 (2012), 825-843.

[18] J. Zhao, Q. Yang, Self-adaptive projection methods for the multiple-sets split feasibility problem, Inverse Probl. 27 (2011), 035009.

[19] J. Zhao, J. Zhang, Q. Yang, A simple projection method for solving the multiple-sets split feasibility problem, Inverse Probl. Sci. Eng. 21 (2013), 537-546.

[20] F. Wang, H. K. Xu, Cyclic algorithms for split feasibility problems in Hilbert spaces, Nonlinear Anal. 74 (2011), $4105-$ 4111.

[21] Y.C. Tang, L.W. Liu, Several iterative algorithms for solving the split common fixed point problem of directed operators with applications, Optimization, 65 (2016), 53-65.

[22] W. Takahashi, Nonlinear functional analysis. Fixed point theory and its applications, Yokohama Publishers, Yokohama, 2000.

[23] K. Goebel, W. A. Kirk, Topics on Metric Fixed Points Theory, Cambridge University Press, Cambridge, 1990.

[24] R.E. Bruck, Properties of fixed point sets of nonexpansive mappings in Banach spaces, Trans. Amer. Math. Soc. 179 (1973), 251-262.

[25] Z.T. Yu, L.J. Lin, C.S. Chuang, A unified study of the split feasible problems with applications, J. Nonlinear Convex Anal. 15 (2014), 605-622.

[26] J.B. Baillon, G. Haddad, Quelques proprietes des operateurs anglebornes et n-cycliquement monotones, Isr. J. Math. 26 (1977), 137-150.

[27] G. Marino, H.K. Xu, A general iterative method for nonexpansive mappings in Hilbert spaces, J. Math. Anal. Appl. 318 (2006), 43-52.

[28] P. Majee, C. Nahak. A hybrid viscosity iterative method with averaged mappings for split equilibrium problems and fixed point problems, Numer Algor. 74 (2017), 609-635.

[29] W. Takahashi, Introduction to Nonlinear and Convex Analysis, Yokohama Publishers, Yokohama, 2009.

[30] H. Zegeye, N. Shahzad, Convergence of Mann's type iteration method for generalized asymptotically nonexpansive mappings, Comput. Math. Appl. 62 (2011), 4007-4014.

[31] P.E. Mainge, A hybrid extragradient-viscosity method for monotone operators and fixed point problems, SIAM J. Control Optim. 47 (2008), 1499-1515.

[32] Z. Xue, H. Zhou, Y.J. Cho, Iterative solutions of nonlinear equations for m-accretive operators in Banach spaces, J. Nonlinear Convex Anal. 1 (2000), 313-320. 